\title{
Towards more evidenced-based therapies for postural tachycardia syndrome and other updates on recent autonomic research
}

\author{
Mitchell G. Miglis ${ }^{1} \cdot$ Srikanth Muppidi ${ }^{1}$
}

Received: 1 March 2021 / Accepted: 2 March 2021 / Published online: 11 March 2021

๑) Springer-Verlag GmbH Germany, part of Springer Nature 2021

Keywords Postural tachycardia syndrome $\cdot$ Autoimmune autonomic ganglionopathy · Ivabradine

\section{Towards more evidenced-based therapies for postural tachycardia syndrome}

When it comes to the treatment of patients with postural tachycardia syndrome (POTS), few pharmacological therapies are evidenced based. While there are clinical trials to support the use of beta blockers such as propranolol and bisoprolol, for example, many therapies are supported by retrospective data. Ivabradine, a cardioselective agent that inhibits the $I_{\text {funny }}$ channel of the sinoatrial node, thereby lowering heart rate without lowering blood pressure, is currently approved by the Food and Drug Administration for patients with systolic heart failure. Its efficacy in POTS has been previously supported by several cases series and retrospective case-control studies, however until now clinical trial data has been lacking.

In their publication "Randomized Trial of Ivabradine in Patients with Hyperadrenergic Postural Orthostatic Tachycardia Syndrome," published in the Journal of the American College of Cardiology [1], Taub et al. report the first clinical trial of ivabradine in POTS. The authors enrolled adult patients ages 18 to 65 with a diagnosis of hyperadrenergic POTS, defined by a standing norepinephrine of $>600 \mathrm{pg} / \mathrm{ml}$ and a heart rate increase of $>30$ beats/min on head-up tilt testing (HUTT). Twenty-six POTS patients were enrolled and randomized to receive ivabradine (2.5-7.5 $\mathrm{mg}$ twice daily) or placebo in a double-blind, placebo-controlled, prospective, parallel-arm design.

All patients were required to undergo a 1-week washout of their POTS medications before the screening visit.

Srikanth Muppidi

muppidis@stanford.edu

1 Stanford Medical Center, Stanford Neurosciences Health Center, 213 Quarry Road, 2nd Floor, Palo Alto, CA 94304, USA
Ivabradine was started at the typical starting dose of $5 \mathrm{mg}$ twice daily and titrated based on tolerability and heart rate response to a 3-min stand test. Two weeks after the titration visit, patients returned for repeat stand testing and norepinephrine measurements, then underwent a 1-week washout period. Patients subsequently crossed over to the other study arm and completed another month of treatment or placebo identical to the first month, with stand testing and norepinephrine levels at completion. Norepinephrine was collected after $15 \mathrm{~min}$ of supine rest and then again after $15 \mathrm{~min}$ of standing, though the authors do not state whether repeat venipuncture or indwelling intravenous catheter (preferred method) was used. Norepinephrine levels were drawn at baseline, at the end of the ivabradine month, and the end of the placebo month. All patients completed the RAND-36 quality of life (QOL) questionnaire. The primary outcome of the study was reduction in heart rate, and the secondary outcome was change in QOL scores.

The average age of patients was slightly older than the typical POTS demographic $(32.5 \pm 11.4$ years $)$. The majority of patients had symptoms 1-3 years prior to POTS diagnosis, and $30 \%$ had a history of syncope, in line with typical prevalence estimates. 19/22 (86\%) of patients were white. Of the 26 patients randomized, 1 dropped out due to other medical issues and 3 dropped out due to side effects of including nausea, fatigue, and visual phosphenes, common side effects of ivabradine. There were no adverse events such as symptomatic bradycardia or hypotension. 6/26 (23\%) patients had their dose down-titrated to $2.5 \mathrm{mg}$ twice daily based on stand testing during the trial.

Ivabradine significantly reduced postural tachycardia (ivabradine $13.1 \pm 8.6 \mathrm{bpm}$ vs. placebo $17.0 \pm 10.4 \mathrm{bpm}$, $p<0.001,95 \%$ CI 0.300-1.250), as well as the average standing HR $(77.9 \pm 9.3 \mathrm{bpm}$ vs. $94.2 \pm 16.2 \mathrm{bpm}, p<0.001$, 95\% CI 0.544-1.58). It is interesting to note that none of these mean HRs are in the tachycardic range (even in 
placebo arm), and that the mean HR improved from patients' baseline on enrollment regardless of study arm they were initially enrolled in (drug or placebo). Compared to placebo, significant improvements were seen in QOL measures of physical functioning $(p=0.008)$ and social functioning $(p=0.021)$ but not in other domains or total RAND-36 scores. Ivabradine did not lower supine $(p=0.316)$ or standing norepinephrine levels $(p=0.076)$; however, when the authors stratified those patients with very high standing norepinephrine levels, they did find a greater magnitude of reduction with ivabradine compared to placebo $(p=0.026)$.

We commend the authors for pursuing higher quality evidence for the treatment of patients with POTS. There are several limitations to this study, however. The first is the small sample size, which may have been underpowered to detect certain treatment effects such as reduction in standing norepinephrine levels. The second is the population selected. It is not clear why the authors studied only the hyperadrenergic population. This significantly limits the evidence-based applications (and possible insurance coverage) of the drug. It is also unclear how the authors drew the norepinephrine samples and how they were analyzed, a critical piece of information if the study is focused on this unique hyperadrenergic cohort. If the repeat venipuncture method was used, the epinephrine values are unreliable. Finally, the HR of patients during the study did not technically meet criteria for POTS, as patients in both treatment and placebo arms had postural changes in HR less than the 30-bpm required for the diagnosis.

Nonetheless, Taub and colleagues are to be commended for their efforts in publishing more rigorous pharmacological data in POTS, and we hope to see future trials enrolling larger numbers of patients in multi-center collaborations to gather more rigorous evidence to support treatments for this condition.

\section{Biomarkers of recovery in autoimmune autonomic ganglionopathy}

Autoimmune autonomic ganglionopathy (AAG), a rare autoimmune autonomic disease caused by autoantibody blockade of the nicotinic ganglionic acetylcholine receptor (gAChR$\mathrm{Ab}$ ), can present with subacute or chronic autonomic failure and is often responsive to immunotherapy. Prior studies evaluating response to treatment predominantly evaluated effects on cardiovascular and sudomotor function or results of autonomic reflex testing (Composite Autonomic Severity Score) with variable evidence of objective benefit, even though patients frequently report improved symptom burden. To document the extent of autonomic recovery after treatment of AAG, Koay et al. recently published their work online [2] titled "Multimodal Biomarkers Quantify Recovery in Autoimmune Autonomic Ganglionopathy" in Annals of Neurology.

For this study, the research team in England recruited 13 patients who were evaluated and treated for AAG (defined by autonomic failure and high serum gAChR antibody levels (>100 pM), evaluated between the years of 2005 and 2019) at their center. This cohort included both patients identified through retrospective chart analysis and prospective recruitment and consisted of seven women and six men with an age range of 21-69 years. All patients were evaluated with multimodal autonomic testing before and after immunotherapy, including cardiovascular autonomic testing (HR variability to deep breathing, Valsalva testing, head-up tilt table testing) with supine and upright norepinephrine levels, pupillary function testing (with infrared pupillometry, and cholinergic and adrenergic supersensitivity testing), sudomotor, lacrimal and salivary testing, and urodynamic studies. Additionally, one patient had skin biopsy with specialized staining to document changes in skin somatic and autonomic denervation and improvement after immunotherapy. All patients had diffuse autonomic failure with orthostatic intolerance, gastrointestinal, genitourinary, sudomotor, secretomotor, and pupillomotor dysfunction. Many patients had other associated autoimmune conditions, five had malignancy and some patients had antecedent infections. Overall disease duration before autonomic testing ranged from 4 months to 18 years. There was no standard immunotherapy protocol for all patients, and most were usually treated with plasma exchange or intravenous immunoglobulin at the onset, followed by corticosteroids and other immunotherapies including mycophenolate, azathioprine, and rituximab.

To assess response to immunotherapy, all patients had repeat multimodal autonomic testing, although the exact duration of treatment before repeat testing was not clearly defined and was likely not uniform. Overall, there was improvement in autonomic function across multiple modalities. There was a clear improvement in orthostatic drop in systolic blood pressure (SBP) and duration of tilt tolerance. Researchers used a new metric called the orthostatic tolerance ratio, which they defined as the ratio of drop in SBP over duration of tilt tolerated, with a higher value representing a greater drop in SBP or shorter duration of tilt tolerance or combination of both. There was clear improvement in the orthostatic intolerance ratio of patients after treatment (33.3 vs. 5.2, $p=0.007$ ). There was also statistical improvement in heart rate response to deep breathing, pupillary light constriction, and saliva production. More importantly, patients noted symptomatic improvement with improved COMASS-31 scores (52 vs. $17, p=0.03$ ). There was no clear improvement in the overall norepinephrine levels, sudomotor, lacrimal, urodynamic metric, and quality of life scores before and after treatments, although there was significant variability between patients. Median ganglionic antibody 
titer reduction was not statistically different. We encourage readers to review the publication for more details on testing and improvement including reviewing the skin biopsy changes with staining for somatic and autonomic fibers after immunotherapy.

As appropriately claimed by the researchers, this remains the most extensively studied cohort of AAG patients with subjective and objective autonomic evaluation before and after treatment. Most patients seem to have an improvement in orthostatic symptoms and improvement in other metrics. Additionally, it seems that other findings of autonomic impairment such as changes on skin biopsy and improvement post-treatment suggest that many of the effects of AAG are physiological and immunotherapy -responsive irrespective of duration of illness. We agree with the authors that efforts should be made to identify ganglionic antibodies in all patients with autonomic failure and all patients with AAG should be offered immunotherapy immediately to maximize the benefit.

While this extensive evaluation of a small cohort of patients in a rare disease is welcome, this publication raises further questions. It seems that the primary improvement in most patients is in the orthostatic domain, with clear improvement in orthostatic metrics both objectively and subjectively. Furthermore, the improvements in autonomic measures such as HR variability with deep breathing might not have a clear clinical correlate. Additionally, a further analysis of treatment response in the pure autoimmune form vs. those diagnosed with malignancy either at onset or later might be valuable to understand the difference in these subtypes of AAG. While patients in this cohort completed multiple modalities of autonomic testing, it was surprising not to see any objective upper and lower GI motility studies, as these can be routinely obtained in all the patients. This might have been useful, as GI-related symptoms can be significant and treatment responsiveness would have been useful to clinical practice. The newly described metric, the orthostatic intolerance ratio, seems to be a reasonable metric as a way to combine the degree of objective SBP drop with patient's subjective tolerance to tilt table testing. Further validation of this metric in both other autoimmune autonomic conditions and non-autoimmune conditions with autonomic failure will be useful. Despite these limitations, the authors should be commended for their efforts for extensive evaluations before and after therapy. This work emphasizes the need to always evaluate for presence of this antibody in patients with autonomic failure, as treatment may significantly improve outcome for patients.

Funding None.

\section{Declarations}

Conflict of interest None.

\section{Reference:}

1. Taub PR, Zadourian A, Lo HC, Ormiston CK, Golshan S, Hsu JC (2021) Randomized trial of ivabradine in patients with hyperadrenergic postural orthostatic tachycardia syndrome. J Am Coll Cardiol [online serial]. Elsevier Inc. 77:861-871. Accessed at: https://pubmed-ncbi-nlm-nih-gov.laneproxy.stanford.edu/33602 468/. Accessed 28 Feb 2021

2. Koay S, Vichayanrat E, Bremner F et al (2021) Multimodal biomarkers quantify recovery in autoimmune autonomic ganglionopathy. Ann Neurol (John Wiley and Sons Inc). https://doi. org/10.1002/ana.26018 (Epub 2021) 